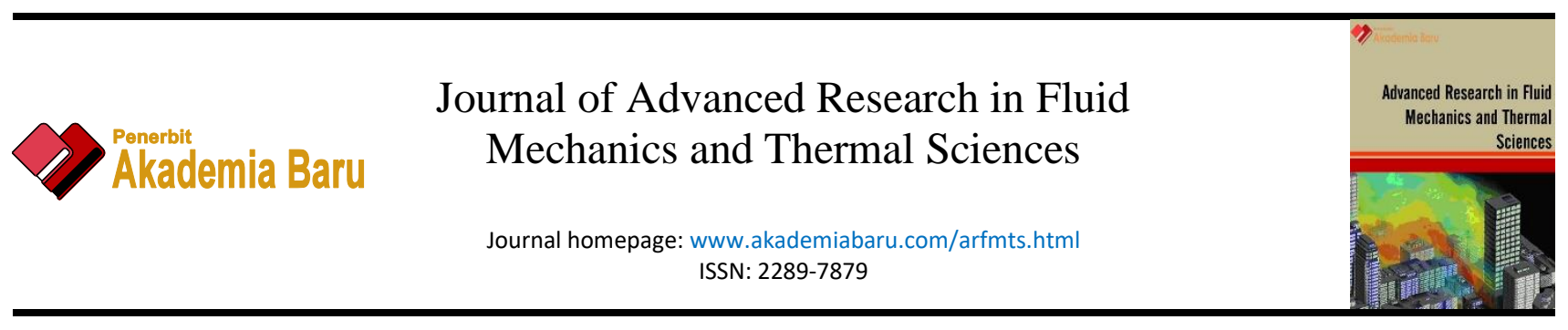

\title{
Computation of Nonlinear Thermoelectric Effects with Adaptive Methods
}

\author{
Aizuddin Mohamed ${ }^{1}$, Razi Abdul-Rahman ${ }^{1, *}$ \\ 1 School of Mechanical Engineering, Universiti Sains Malaysia, 14300 Nibong Tebal, Pulau Pinang, Malaysia
}

\section{ARTICLE INFO \\ ABSTRACT}

\section{Article history:}

Received 27 May 2020

Received in revised form 5 September 2020

Accepted 10 September 2020

Available online 25 October 2020

Keywords:

Thermoelectricity; Nonlinear solvers; Finite Element Method
An implementation for a fully automatic adaptive finite element method (AFEM) for computation of nonlinear thermoelectric problems in three dimensions is presented. Adaptivity of the nonlinear solvers is based on the well-established $h p$-adaptivity where the mesh refinement and the polynomial order of elements are methodically controlled to reduce the discretization errors of the coupled field variables temperature and electric potential. A single mesh is used for both fields and the nonlinear coupling of temperature and electric potential is accounted in the computation of a posteriori error estimate where the residuals are computed elementwise. Mesh refinements are implemented for tetrahedral mesh such that conformity of elements with neighboring elements is preserved. Multiple nonlinear solution steps are assessed including variations of the fixed-point method with Anderson acceleration algorithms. The Barzilai-Borwein algorithm to optimize the nonlinear solution steps are also assessed. Promising results have been observed where all the nonlinear methods show the same accuracy with the tendency of approaching convergence with more elements refining. Anderson acceleration is the most efficient among the nonlinear solvers studied where its total computing time is less than half of the more conventional fixed-point iteration.

\section{Introduction}

Most computations of complex physical phenomena today use mathematical schemes by which models with a finite number of unknowns are built. As the system continues to grow in complexity, an analyst may have to deal with exponential increase in the computational effort needed to solve the problem. Adaptive Finite Element Method (AFEM) is an improvement of the mature conventional FEM that optimizes the selection of parameters so that better accuracy can be achieved with less computational effort. The most common of this technique is the $h p$-adaptive strategy by which $h$ stands for mesh refining while $p$ stands for polynomial element refining [1]. Both are carefully

\footnotetext{
* Corresponding author.

E-mail address: mearahman@usm.my
}

https://doi.org/10.37934/arfmts.76.3.7584 
controlled throughout the iterative adaptation process. Coarse mesh with the lowest order of elements is used as the initial mesh for the process of adaptation and progresses to a combination of a finer mesh and higher order of elements in selected parts of the computational domain. The control process is automated by which it recognises high residual error of an element from $a$ posteriori error computation and reduces the error element-wise after each level of adaptation until convergence.

Although adaptive methods are deemed a mature finite element scheme for linear problems, nonlinearities pose exceptional challenges [2]. In this paper, the efficacy of AFEM for solving nonlinear thermoelectric problem in three dimensions is assessed. Specifically, the nonlinearity that arises due to the electric and thermal fluxes being mutually dependent [1]. Dealing with a rapid growth of degrees of freedom (DOF) is challenging since the nonlinear system matrix gets larger and becomes less sparse due to the presence of high order finite elements. The work by Babuška and Guo [3] has established the fact that an optimal selection of parameters helps increase the convergence rate exponentially in a single field problem. However, achieving optimal convergence rates for nonlinear multi-physics remains an open problem. This paper attempts numerical studies on various nonlinear solvers to assess the accuracy and efficiency of the algorithms in the $h p$-adaptive solutions. All the studies were performed with the in-house code PolyDE that is capable to solve with fully automatic adaptive procedures in three dimensions using tetrahedral elements [4].

\section{Methodology}

\subsection{General Multiphysics Equations}

In PolyDE, a generalized set of coupled partial differential equation (PDE) for a multiphysics problem is implemented such that the PDE forms can account a wide range of coupling phenomena in physics without changing the structure of the PDEs. In the specific case of thermoelectricity, a single set of PDEs completely describes the mutual coupling of temperature and potential difference. This monotholic approach allows for a fully automatic adaptivity in a single mesh for all the unknown fields. The effects resulting from the interaction with the other field quantities can also be included [4]. The generalized PDE reads

$-\nabla \cdot\left(v_{i j} \nabla u+\gamma_{i j} u\right)+\beta_{i j} \nabla u+\eta_{i j} u=f_{i}+\nabla \cdot \mathrm{g}_{i}$

where in thermoelectricity $u$ is either temperature or electric potential. The coefficient tensors $v, \gamma$, $\beta$ and $\alpha$ describe corresponding material properties. The right hand-side terms may comprise of a scalar field $f_{i}$ or divergence of a vector field $g_{i}$. Although the approach taken for solving specific problems differs, a new multi-physics mode can be introduced into the code without the need of generating another system of PDEs.

\subsection{The Galerkin Formulation for Nonlinear Thermoelectricity}

The implementation of the finite element procedures for thermoelectricity analysis is based largely on Antonova and Looman [5]. However, unlike the formulation in research of Antonova and Looman [5], the coupled equations are made symmetric as described by Vokas and Kasper [4]. Restricting the analysis to steady-state, the coupling between the temperature field and electric potential field in a thermoelectric material is summarized in terms of thermal heat flux $q$ and current density J as 
$\mathrm{q}=T \alpha \mathrm{J}-\lambda \nabla T$

$\mathrm{J}=\sigma \mathrm{E}-\alpha \nabla T$

where

$T$ = temperature (K),

$\alpha=$ Seebeck coefficient $(\mathrm{V} / \mathrm{K})$,

$\lambda=$ thermal conductivity $\left(\mathrm{Wm}^{-1} \mathrm{~K}^{-1}\right)$,

$\sigma=$ electric conductivity $(\mathrm{S} / \mathrm{m})$, and

$E=$ electric field strength $(\mathrm{V} / \mathrm{m})$.

Since in this case the electric field can be expressed in terms of the electric potential $\phi$,

$\mathrm{E}=-\nabla \phi$

in terms of temperature and electric potential, the coupled equations then read

$-\nabla \cdot(\Pi \sigma \nabla \phi)-\nabla \cdot(\lambda \nabla T)=Q$

$\nabla \cdot(\sigma \alpha \nabla T)+\nabla \cdot(\sigma \nabla \phi)=0$

Eq. (5) and Eq. (6) can be recast into the general coupled Eq. (1) in which the material coefficients and the load vectors are defined for thermoelectricity. Observing Eq. (5), the heat generation comes from different sources: external sources or internal sources in the form of electric power generated as Joule heating or as work against the Seebeck effect. To simplify the current study, only the work against the Seebeck effect is considered. Inclusion of this effect means the right-hand side of Eq. (5) is in dependence of $\phi$, and thus the system of PDEs is now nonlinear.

Nonlinearity is also the result of accounting for the Thomson effect. Although it is considered the third effect in thermoelectricity, it can also be seen as the consequence of temperature dependency of the Seebeck effect. The Thomson coefficient $\tau$ with unit $\mathrm{V} / \mathrm{K}$ reads

$\tau=T \frac{d \alpha}{d T}$

from which it implies the absence of the Thomson effect in the case of constant Seebeck coefficient. For a more realistic modeling of thermoelectric materials, accounting for the temperature variations leads to Eq. (1) having nonlinear material coefficients. Nevertheless, Thomson effect is ignored in this study.

Following the standard Galerkin method, the discrete solutions for temperature and electric potential for the finite element space $\bar{T}$ and $\bar{\phi}$ are

$\bar{T}=\sum_{n=1}^{m} \xi_{n} \Phi_{1, n}$

$\bar{\phi}=\sum_{n=1}^{m} \xi_{n} \Phi_{2, n}$

where $\Phi_{1, n}$ and $\Phi_{2, n}$ are the respective degrees of freedom and $\xi_{n}$ are the basis functions defined in a tetrahedron. Transforming the strong forms of Eq. (5) into their respective weak form in the usual manner results in nonsymmetric system matrices. Here the procedure follows the work of Vokas and 
Kasper [4] where by using the Onsager reciprocity theorem, transformation to the weak forms results in symmetric system matrices. The weak forms are summarized as

$\int_{\Omega} \sum_{n=1}^{m}-\left(\alpha^{2} \sigma+\frac{\lambda}{T_{0}}\right) \nabla \xi_{n} \nabla \xi_{d} \Phi_{1, n}-\alpha \sigma \nabla \xi_{n} \nabla \xi_{d} \Phi_{2, n} d \Omega=\int_{\Omega} \sum_{n=1}^{m} \xi_{n} Q d \Omega \quad d=1,2, \cdots, m$

$\int_{\Omega} \sum_{n=1}^{m}-\alpha \sigma \nabla \xi_{n} \nabla \xi_{d} \Phi_{1, n}-\sigma \nabla \xi_{n} \nabla \xi_{d} \Phi_{2, n} d \Omega=0 \quad d=1,2, \cdots, m$

where $T_{0}$ is the reference temperature. Combining Eq. (10) and Eq. (11) leads to a single system matrix $\mathrm{Ka}=\mathrm{b}$ such that

$\left[\begin{array}{ll}\mathrm{K}_{11} & \mathrm{~K}_{12} \\ \mathrm{~K}_{21} & \mathrm{~K}_{22}\end{array}\right]\left[\begin{array}{l}\Phi_{1, n} \\ \Phi_{2, n}\end{array}\right]=\left[\begin{array}{c}\mathrm{b}_{1, n} \\ 0\end{array}\right]$

The element stiffness matrix $\mathrm{K}_{I J}$ composes of

$\mathrm{K}_{11}=\int_{\Omega} \sum_{n=1}^{m}-\left(\alpha^{2} \sigma+\frac{\lambda}{T_{0}}\right) \nabla \xi_{n} \nabla \xi_{d} d \Omega \quad d=\{1,2, \cdots, m\}$,

$\mathrm{K}_{12}=\mathrm{K}_{21}=\int_{\Omega} \sum_{n=1}^{m} \alpha \sigma \nabla \xi_{n} \nabla \xi_{d} d \Omega \quad d=\{1,2, \cdots, m\}$,

$\mathrm{K}_{22}=\int_{\Omega} \sum_{n=1}^{m}-\sigma \nabla \xi_{n} \nabla \xi_{d} d \Omega \quad d=\{1,2, \cdots, m\}$,

The element load vector associated with Eq. (5) relates the work against the Seebeck effect. The electromotive field that is created from the Seebeck effect is

$\mathrm{E}_{\mathrm{emf}}=-\alpha \nabla T$,

so that the power as work against this field comes from the Peltier effect such that

$P=\mathrm{J} \cdot \Pi \cdot \nabla T$

The load vector is then

$\mathrm{b}_{1, n}=\int_{\Omega} \sum_{n=1}^{m} \nabla \xi_{n} \cdot \Pi \cdot \mathrm{J} d \Omega$

The load vector is in dependence of $\Phi_{2, n}$ due to the presence of J, which renders the system matrix to be nonlinear, and thus the computation involves searching for the zeros of

$\left[\begin{array}{ll}\mathrm{K}_{11} & \mathrm{~K}_{12} \\ \mathrm{~K}_{21} & \mathrm{~K}_{22}\end{array}\right]\left[\begin{array}{l}\Phi_{1, n} \\ \Phi_{2, n}\end{array}\right]-\left[\begin{array}{c}\mathrm{b}_{1, n}\left(\Phi_{2, n}\right) \\ 0\end{array}\right]=0$

which is essentially a nonlinear problem

$\mathrm{F}\left(\Phi_{i, n}\right)=0 \quad x=1,2$ 


\subsection{Hp-Finite Element Solver}

Solving the nonlinear form of Eq. (1) involves roots finding or for the case of its discrete counterparts, finding the solution for $F(\Phi)=0$. Numerical solvers for nonlinear equations involve iterations that produce approximations of the roots. Hence, convergence of solutions is of most interest in nonlinear schemes. Selected iterative schemes for nonlinear equations which is the interest of this work will be assessed to find more suitable algorithms in terms of accuracy and efficiency for the nonlinear thermoelectric problem.

\subsubsection{Fixed-point iteration}

One of the main tools for solving a nonlinear problem is the fixed-point iteration method. Fixedpoint method is a well-known open method that uses a general strategy of successive substitutions based on iteration formulas requiring only a starting point or pair of values. The most basic iterative scheme reads

$x_{i+1}=g\left(x_{i}\right)$

where the iteration index $i=0,1,2, \ldots$ and $x_{0}$ is the initial estimate of the root as seen in Figure 1 . This method is preferred because of the simplicity and flexibility to choose the form of $g(x)$. Nevertheless, there are cases where the iterations do not always converge for a chosen $g(x)$. To ensure convergence of the iterative scheme for the interval containing the root, $|g(x)|<1$ condition must be satisfied [6]. If otherwise, the solution will diverge.

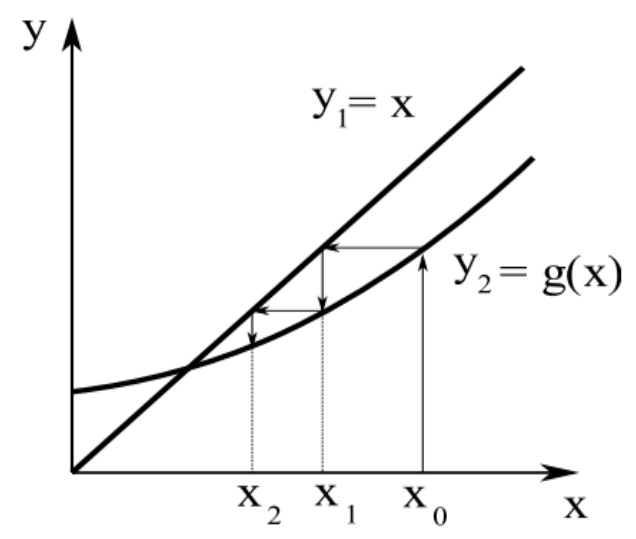

Fig. 1. Iterative convergence of fixedpoint method [6]

\subsubsection{Anderson acceleration of fixed-point iteration}

The main drawback of the fixed-point scheme is its very slow rate of convergence [7]. Failure or improper behavior of convergence makes this method not a primary choice of solvers. The work by Anderson [8] is a significant success since it shows that convergence is faster with less iteration counts. There are two types of Anderson acceleration, i.e., Anderson relaxation and Anderson extrapolation method. Both of these techniques are not only applicable to fixed-point method but they are also applicable to other iterative solvers such as the Jacobi method $[9,10]$. Generally, Anderson acceleration iterative scheme is as follows 
$x^{k+1}=\sum_{j=0}^{m-1} \alpha_{j+1} x^{k-j}$

The coefficients $\left\{\alpha_{1}, \ldots, \alpha_{m}\right\}$ are selected by solving the minimization problem

$\min _{\text {alpha } a \in \mathbb{R}^{m}}\left\|\sum_{j=0}^{m-1} \alpha_{j+1} e^{k-j}\right\|^{2}, \sum_{j=0}^{m} \alpha_{j}=1 \quad(j \in\{0, \ldots, m-1\})$

The idea is to select linear combinations of $m$ previous iterates in such a way that the residual decreases as much as possible. For the extrapolation method the only change is to $j \in\{0, \ldots, m+1\}$.

\subsubsection{Barzilai-Borwein method (BB)}

The Barzilai and Borwein (BB) method is a variation of steepest descent method originally proposed for solving a minimization problem of a typical quadratic function

$\min f(x)=\frac{1}{2} x^{T} A x-b^{T} x$

where $A$ is a real symmetric positive definite matrix and $b$ is constant. The BB method was proven to yield significantly faster convergence than the classical gradient method for solution of linear systems while requiring less computer memory. Due to this appeal, significant efforts were geared toward application of the BB method for solving nonlinear systems [11]. In the current implementation, the updated solution $x_{k+1}$ uses the scaling parameter $\alpha_{k}$ in

$x_{k+1}=x_{k}+\alpha_{k} g\left(x_{k}\right)$

where $\alpha_{k}$ is evaluated according to the BB method as in research of Calderón et al., [10]. $g\left(x_{k}\right)$ relates to a search direction.

\subsection{AFEM Modeling Strategies}

The present implementation of the $h p$-adaptive FEM procedure was described by Kamaludin and Abdul-Rahman [12] and Kamaludin [13] showing how presence of singularity in the domain is resolved with the refinement of tetrahedral mesh. The mesh refinement preserves element connectivity unlike in other implementations for example in the research by Solin et al., [14]. In this test case, to avoid any geometric singularity, a simple model of thermoelectric leg is constructed as in Figure 2. The test case is similar to that of Jaegle [15]. Boundary conditions are prescribed with both fields' temperature and electric potential for this simulation. A temperature of $273 \mathrm{~K}$ is prescribed on the bottom plate and homogenous Neumann at the top plate. The rest of the surface is treated adiabatically. For the electric potential, Neumann boundary condition is prescribed at the top plate while the bottom plate is treated as ground. Both the top and bottom plates material is made up of copper, while bulk material is made up of bismuth telluride, $\mathrm{Bi}_{2} \mathrm{Te}_{3}$. The threedimensional case is used in modeling the thermoelectric problem to provide sufficiently large system matrix for assessing the nonlinear solvers with $h p$-adaptivity. 


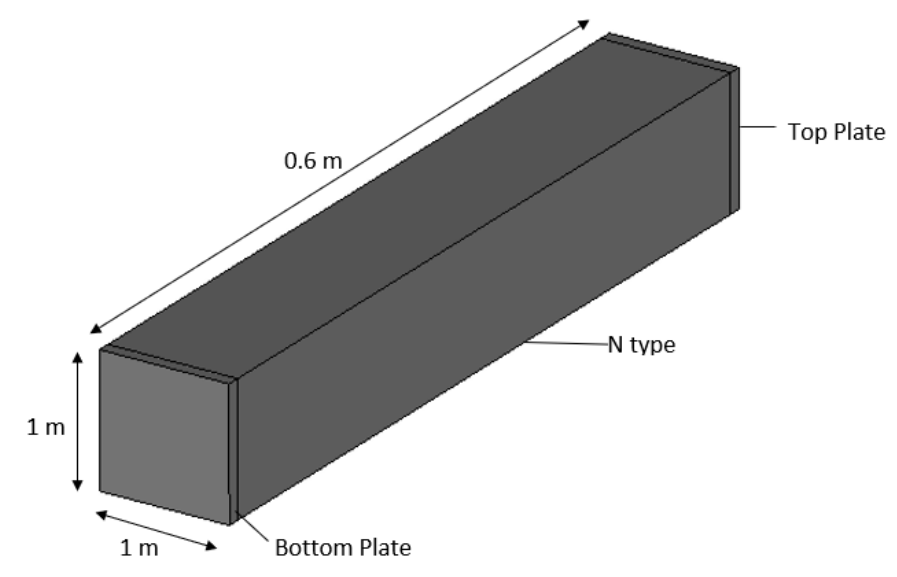

Fig. 2. Thermoelectric model of $n$ type semiconductor

\section{Result and Discussion}

The automated $h p$-adaptive process of the thermoelectric solution shows a significant increase in the number of elements, with the initial mesh of 989 elements terminated with 10,364 elements as seen in Figure 3. The adaptive process undergoes 6 steps of adaptive processes with generally decreasing energy norm error as listed in Table 1.

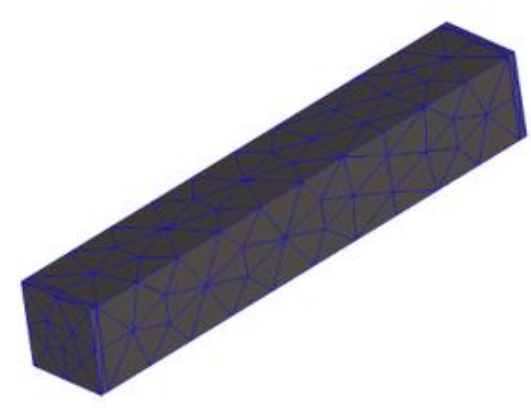

989 elements

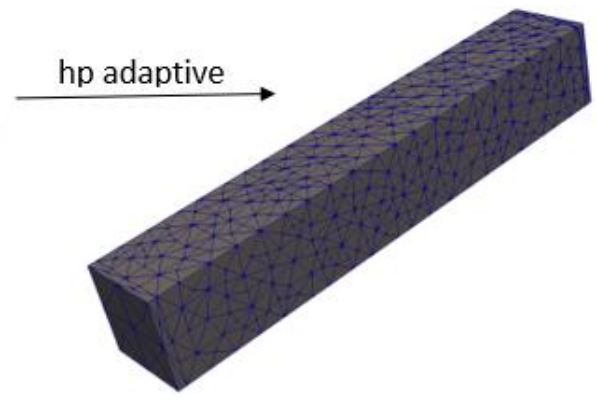

10364 elements

Fig. 3. $h p$-Adaptivity in the nonlinear thermoelectric computation

Table 1

Multi-step adaptations for the nonlinear solutions of temperature $T$ and electric potential $V$ fields

\begin{tabular}{llll}
\hline Adaptation steps & NDOF & Energy norm error of $T$ & Energy norm error of $V$ \\
\hline 0 & 382 & $4.23 \times 10^{-4}$ & $8.52 \times 10^{-8}$ \\
1 & 524 & $3.04 \times 10^{-4}$ & $5.74 \times 10^{-8}$ \\
2 & 832 & $2.34 \times 10^{-4}$ & $4.14 \times 10^{-8}$ \\
3 & 1297 & $1.75 \times 10^{-4}$ & $1.43 \times 10^{-6}$ \\
4 & 1938 & $1.36 \times 10^{-4}$ & $1.39 \times 10^{-7}$ \\
5 & 2961 & $1.07 \times 10^{-4}$ & $6.20 \times 10^{-7}$ \\
6 & 4564 & $8.24 \times 10^{-5}$ & $3.29 \times 10^{-7}$ \\
\hline
\end{tabular}

\subsection{Accuracy of hp-FEM Nonlinear Solution}

According to Zeng et al., [16], nonlinear iterative solvers have varying degrees of accuracy, computational costs in terms of time and memory, and convergence rates. The quality of each method depends upon how close each iteration is in approaching $f(x)=0$. To compare the 
accuracy of the nonlinear solvers used in this study, the L2 norms of the deviations in the results with all the solvers were computed. The $L 2$ norm of the deviations were evaluated with respect to the fixed-point method since it was observed that it yields the best results as shown in Figure 4 and Figure 5 in the computations for the temperature and electric potential. The fields distributions in the middle of the thermoelectric leg along its length clearly show the nonlinear behavior of the material. The nonlinear results are more apparent in the temperature compared to the voltage. The temperature varies nonlinearly from $212 \mathrm{~K}$ to $273 \mathrm{~K}$. The nonlinear distribution of the voltage is less apparent as it only varies between 0 and $0.049 \mathrm{~V}$.

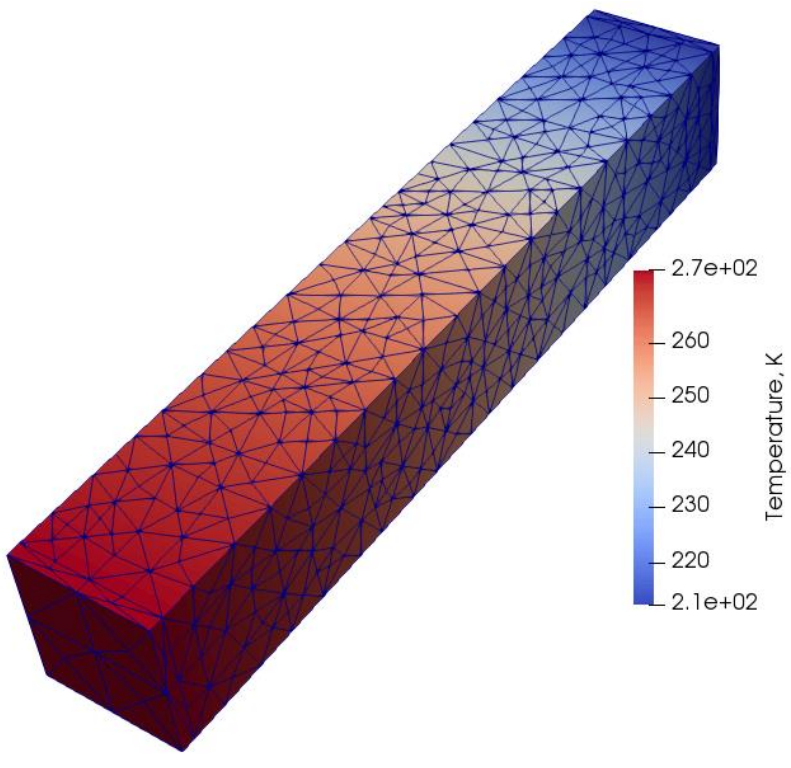

(a)

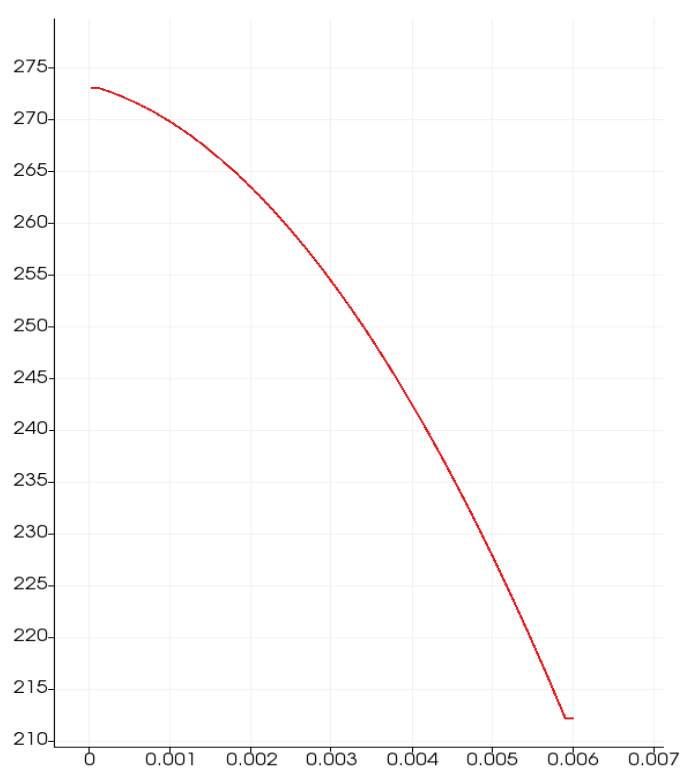

(b)

Fig. 4. (a) Temperature field of thermoelectric model, (b) Plot of temperature field

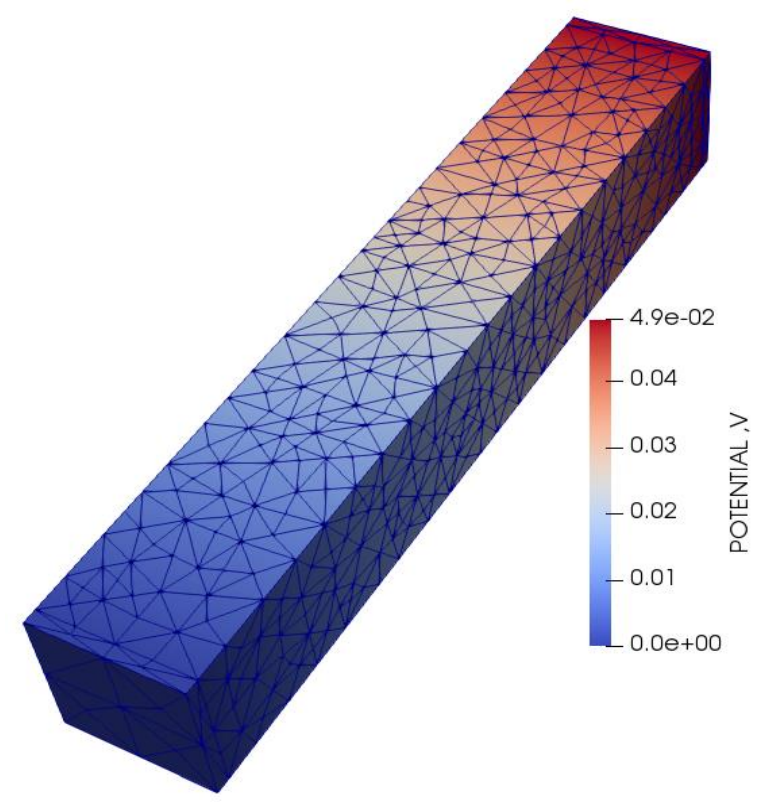

(a)

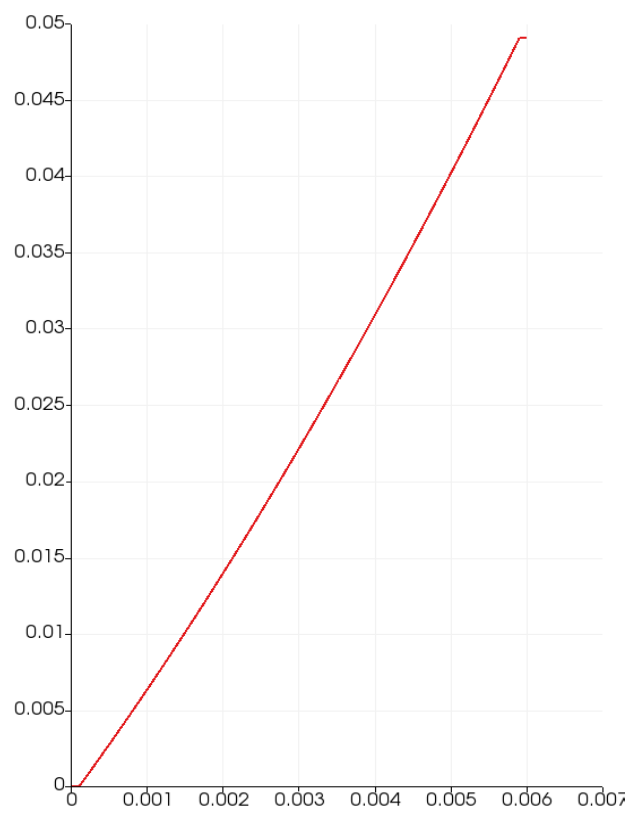

(b)

Fig. 5. (a) Voltage potential field of thermoelectric model, (b) Plot of electric potential field 
For the same adaptation tolerance, all the four methods achieve convergence with final NDOF of 4564. From Table 2, the BB method shows the largest error from the reference fixed-point solver at 0.83 while the errors for both Anderson relaxation and extrapolation are the same at 0.43 . The voltage norm shows no difference between the 4 solvers.

Table 2

Comparison of accuracy of iterative methods

\begin{tabular}{llll}
\hline Methods & Final NDOF & $\begin{array}{l}\text { L2 norm for } \\
\text { Temperature }\end{array}$ & $\begin{array}{l}\text { L2 norm for } \\
\text { Voltage }\end{array}$ \\
\hline Fixed point & 4564 & - & - \\
Anderson relaxation & 4564 & 0.431437 & - \\
$\begin{array}{l}\text { Anderson } \\
\text { extrapolation }\end{array}$ & 4564 & 0.431437 & - \\
Barzilai-Borwein & 4564 & 0.830156 & - \\
\hline
\end{tabular}

\subsection{Efficiency of hp-FEM Nonlinear Solver}

The total cost of solving the problem depends on both the cost per iteration and the number of iterations required for convergence [17]. Table 3 shows that the highest number of iterations is 197 for the fixed-point method while the lowest iteration is obtained with both Anderson methods. The total cost for the fixed-point method shows the highest value with 229 seconds among all the others. Both Anderson acceleration methods show the same value of total cost of 104 seconds which is the lowest among the assessed methods.

Table 3

Comparison of efficiency of iterative methods

\begin{tabular}{llll}
\hline Methods & Number of iterations & Cost per iteration (s) & Total Cost (s) \\
\hline Fixed point & 197 & 1.16 & 229 \\
Anderson relaxation & 90 & 1.15 & 104 \\
Anderson extrapolation & 90 & 1.15 & 104 \\
Barzilai-Borwein & 178 & 1.16 & 207 \\
\hline
\end{tabular}

\section{Conclusion}

In summary, all the nonlinear solvers assessed in this study can achieve the same accuracy in multiphysics computation whereby the Anderson acceleration algorithms show a good potential in terms of efficiency. The fully automated $h p$-adaptive procedure for nonlinear problem can be made more efficient for a faster convergence by emphasizing in mesh refining rather than increasing the element order. To enable higher element orders, more sophisticated nonlinear schemes may be necessary.

\section{Acknowledgement}

This research was funded by a grant from Ministry of Higher Education of Malaysia (FRGS Grant FRGS/1/2017/TK03/USM/03/4). 


\section{References}

[1] Pérez-Aparicio, José L., R. L. Taylor, and Daniel Gavela. "Finite element analysis of nonlinear fully coupled thermoelectric materials." Computational Mechanics 40, no. 1 (2007): 35-45.

https://doi.org/10.1007/s00466-006-0080-7

[2] Demkowicz, Leszek, Jason Kurtz, David Pardo, Maciek Paszenski, Waldemar Rachowicz, and Adam Zdunek. Computing with hp-Adaptive Finite Elements: Volume II Frontiers: Three Dimensional Elliptic and Maxwell Problems with Applications. CRC Press, 2007.

https://doi.org/10.1201/9781420011692

[3] Babuška, Ivo, and B. Q. Guo. "The $h, p$ and hp version of the finite element method; basis theory and applications." Advances in Engineering Software 15, no. 3-4 (1992): 159-174.

https://doi.org/10.1016/0965-9978(92)90097-Y

[4] Vokas, Christos, and Manfred Kasper. "Adaptation in coupled problems." COMPEL: Int J for Computation and Maths. in Electrical and Electronic Eng. 29, no. 6 (2010): 1626-1641.

https://doi.org/10.1108/03321641011078698

[5] Antonova, Elena E., and David C. Looman. "Finite elements for thermoelectric device analysis in ANSYS." In ICT 2005. 24th International Conference on Thermoelectrics, 2005., pp. 215-218. IEEE, 2005. https://doi.org/10.1109/ICT.2005.1519922

[6] Dlala, Emad, and Antero Arkkio. "Analysis of the convergence of the fixed-point method used for solving nonlinear rotational magnetic field problems." IEEE Transactions on Magnetics 44, no. 4 (2008): 473-478.

https://doi.org/10.1109/TMAG.2007.914888

[7] Shevchenko, Igor V. "The fixed point iteration and newton's methods for the nonlinear wave equation." In AIP Conference Proceedings, vol. 1048, no. 1, pp. 482-485. American Institute of Physics, 2008. https://doi.org/10.1063/1.2990968

[8] Anderson, Donald G. "Iterative procedures for nonlinear integral equations." Journal of the ACM (JACM) 12, no. 4 (1965): 547-560.

https://doi.org/10.1145/321296.321305

[9] Pratapa, Phanisri P., Phanish Suryanarayana, and John E. Pask. "Anderson acceleration of the Jacobi iterative method: An efficient alternative to Krylov methods for large, sparse linear systems." Journal of Computational Physics 306 (2016): 43-54. https://doi.org/10.1016/i.jcp.2015.11.018

[10] Calderón, Wilson Rodríguez, and Myriam Rocío Pallares-Muñoz. "Formulations to overcome the divergence of iterative method of fixed-point in nonlinear equations solution." Tecnura 19, no. 44 (2015): 191-199. https://doi.org/10.14483/udistrital.jour.tecnura.2015.2.a14

[11] Mohammad, Hassan. "Barzilai-Borwein-Like Method for Solving Large-Scale Non-Linear Systems of Equations." Journal of the Nigerian Mathematical Society 36, no. 1 (2017): 71-83.

[12] Kamaludin, Siti Sarah, and Razi Abdul-Rahman. "Fully automatic adaptive finite element procedure for multiphysics problems in three dimensions." In AIP Conference Proceedings, vol. 1605, no. 1, pp. 535-539. American Institute of Physics, 2014.

https://doi.org/10.1063/1.4887645

[13] Kamaludin, Siti Sarah. "The hp-Adaptive Finite Element Method In Three Dimensional Multiphysics." Master's Thesis, Universiti Sains Malaysia, 2013.

[14] Solin, Pavel, Lenka Dubcova, Jakub Cerveny, and Ivo Dolezel. "Adaptive hp-FEM with arbitrary-level hanging nodes for Maxwell's equations." Adv. Appl. Math. Mech 2, no. 4 (2010): 518-532. https://doi.org/10.4208/aamm.10-m1012

[15] Jaegle, Martin. "Multiphysics simulation of thermoelectric systems-modeling of Peltier-cooling and thermoelectric generation." In COMSOL Conference 2008 Hannover, no. 6. 2008.

[16] Zeng, Zhezhao, Dongmei Lin, and Lulu Zheng. "Novel Algorithms Solving Nonlinear Equation in Very Large Scope." Journal of Computers 5, no. 4 (2010): 607-616. https://doi.org/10.4304/icp.5.4.606-613

[17] Rosseel, Eveline, and Stefan Vandewalle. "Iterative solvers for the stochastic finite element method." SIAM Journal on Scientific Computing 32, no. 1 (2010): 372-397.

https://doi.org/10.1137/080727026 